\title{
Determinação da densidade linear de carga de um canudinho de refresco (um experimento simples e de materiais acessíveis) ${ }^{+*}$
}

\author{
Carlos Eduardo Laburú ${ }^{1}$ \\ Osmar Henrique Moura da Silva ${ }^{2}$ \\ Departamento de Física - Universidade Estadual de Londrina \\ Paulo Sérgio de Camargo Filho ${ }^{3}$ \\ Universidade Federal Tecnológica do Paraná \\ Londrina - PR
}

\section{Resumo}

Apresenta-se um experimento simples para medir a densidade linear de carga de um bastão com objetivo de aplicar conceitos ensinados de eletricidade para graduandos e alunos do nível médio de ensino. Devido à facilidade de aquisição do material, montagem e procedimento empírico, a atividade pode ser realizada em sala de aula na forma de exercício experimental, quando do desenvolvimento da teoria, ou no espaço usual para somente resolução de exercícios para aplicação da teoria, situação que a torna uma atividade diferenciada posto conjugar exercício tradicional e necessidade de obter medições reais. O bastão para a medida da densidade linear é constituído de um canudinho de refresco eletrizado mediante processo de atrito.

Palavras-chave: Experimento Simples; Física; Densidade Linear de Carga; Ensino Médio e Superior.

\begin{abstract}
A simple experiment is presented to measure the linear charge density of a rod in order to apply taught concepts of electricity to undergraduates and students of the secondary level of education. Due to the ease of material acquisition, assembly and empirical procedure, the activity can be
\end{abstract}

\footnotetext{
${ }^{+}$Determination of the linear charge density of a soda straw (a simple experiment with accessible materials)

* Recebido: fevereiro de 2017. Aceito: novembro de 2017.

${ }^{1}$ E-mail: laburu@uel.br; ${ }^{2}$ E-mail: osmarh@uel.br; ${ }^{3}$ E-mail: paulocarmargo@utfpr.edu.pr
} 
carried out in the classroom as an experimental exercise during the development of the theory, or at the usual time only to solve exercises for theory application. The latter situation makes it a differentiated activity because it combines traditional exercises with the need to get real measurements. The rod used to measure the linear charge density consists of a soda straw electrified by process of friction.

Keywords: Simple Experiment; Physics; Linear Charge Density; High School and College.

\section{Introdução}

O trabalho propõe um experimento simples e de baixo custo que permite alunos de graduação, e mesmo alunos de nível médio de ensino, aplicar conceitos de eletricidade. $\mathrm{O}$ artigo mostra como determinar empiricamente a densidade linear elétrica da superfície de uma linha de cargas de um canudinho de refresco carregado pelo processo de atrito com um papel. Esse conceito físico, entre outros correlacionados com o tema, é deveras abstrato, dificultando a aprendizagem haja vista que costumam ser tratados com os estudantes apenas no formato de exercícios após apresentação teórica.

A propositura da atividade experimental situa-se dentro do que Millar (1987) qualifica de expor um paradigma, de viés epistemológico kuhniano, distinguindo-se de orientações educacionais fundamentadas no teste de hipóteses, de viés epistemológico popperiano (LABURÚ; SILVA; FORÇA, 2012). Millar (1987) afirma que o expor um paradigma significa fazer com que raciocínios, ações e observações dos estudantes sejam guiados por conhecimentos prévios baseados em teorias ensinadas e que devem ser aplicadas.

Em paralelo com esse viés epistemológico especificado da atividade, coloca-se uma componente de ordem cognitivista. Por esta, investigadores com preocupação em transpor estudos do campo da semiótica para a educação científica, mostram que atividades experimentais oportunizam para os estudantes modos de representação diferenciados dos empregados no tratamento exclusivo de resolução de exercícios (LABURÚ; SILVA, 2011). O modo de representação 3-D (PRAIN; WALDRIP, 2006) que utiliza gestos (KENDON, 2005; KIM; ROTH; THOM, 2011) e gesticulações (LABURÚ; SILVA; ZÔMPERO, 2015) complementam e ampliam os modos representacionais, o que permite aumentar o potencial de apropriação significativa do conteúdo. Isto porque a ação sobre os objetos toma parte essencial do processamento cognitivo. Eles não são simplesmente enfeites de expressão, mas partes integrantes da própria linguagem. Linguagem e Gesto oferecem ampla gama de abordagens teóricas, com ênfase não apenas em descrições comportamentais, mas também sobre a formação dos processos cognitivos subjacentes (McNEILL, 2005).

Em razão da facilidade de obtenção do material utilizado e da realização experimental, a atividade experimental sugerida prescinde de um laboratório, sendo capaz de ser realiza- 
da em sala de aula comum durante exercícios de aplicação do estudado. Como consequência da simplicidade do equipamento e procedimentos experimentais, a atividade com essas características é, ademais, didaticamente favorável para a apropriação dos conteúdos envolvidos, pois o aluno tem a conveniência de melhor se concentrar nos conceitos enfocados em comparação com uma preocupação em entender o funcionamento do equipamento, sua manipulação e retirada de dados.

\section{Equipamentos e procedimento experimental}

\section{Material necessário}

- Canudinhos plásticos de refresco;

- Meia de nylon feminina;

- Duas pequenas miçangas (diâmetro de $4 \mathrm{~mm}$ cada);

- Transferidor;

- Copinho de café;

- Gesso;

- Papel toalha ou higiênico;

- Colchete latonado;

- Celular.

\section{Montagem e operação do equipamento}

O equipamento consiste de dois suportes feitos com copinhos plásticos para café, gesso e colchete, conforme detalhes no volume desta revista em Laburú; Silva; Barros (2008). Em cada suporte é pendurado uma miçanga a um fio de nylon retirado (desfiado) de meia calça feminina de modo a formar um pêndulo simples, que, ao serem as miçangas carregadas por contato com o canudinho de refresco eletrizado e assim aproximadas, configuram um pêndulo duplo eletrizado ${ }^{2}$. Caso este em que as miçangas carregadas mantêm-se distantes devido à repulsão mútua por força elétrica atuante (Fig. 1A). Para eletrizar as miçangas, primeiramente neutras e separadas em seus suportes, realiza-se um toque em cada uma com o canudinho eletrizado. Em razão dos materiais empregados serem idênticos, as cargas das miçangas apresentam mesmos sinais de cargas e distribuídas em suas superfícies, ocasionando a repulsão. Em razão de possuírem mesma capacitância, a quantidade de carga elétrica de cada miçanga é semelhante (ver subseção abaixo). Logo, essa repulsão inicial entre as miçangas carregadas produz um ângulo $(\theta)$ que deve ser medido, assim como o comprimento do pêndulo $(l)$, para que seja determinado a carga elétrica $(Q)$ das miçangas, necessitando também a medida de seus pesos $(P)$ (cf. subseção cálculos e medidas). O experimento termina ao se po-

\footnotetext{
${ }^{2}$ Sugere-se aqui a eletrização separada de dois pêndulos simples em razão dos testes mostrarem ser esta realização experimental mais fácil, aproximando-os cautelosamente, em comparação à de um único suporte que configure um pêndulo duplo, devido ao frequente entrelaçamento dos fios de meia calça que esta última ocasiona.
} 
sicionar o canudinho eletrizado entre as miçangas carregadas e tirar a nova medida do novo ângulo $\left(\theta^{\prime}\right)$ entre elas, conforme a Fig. 1B (cf. subseção cálculos e medidas).

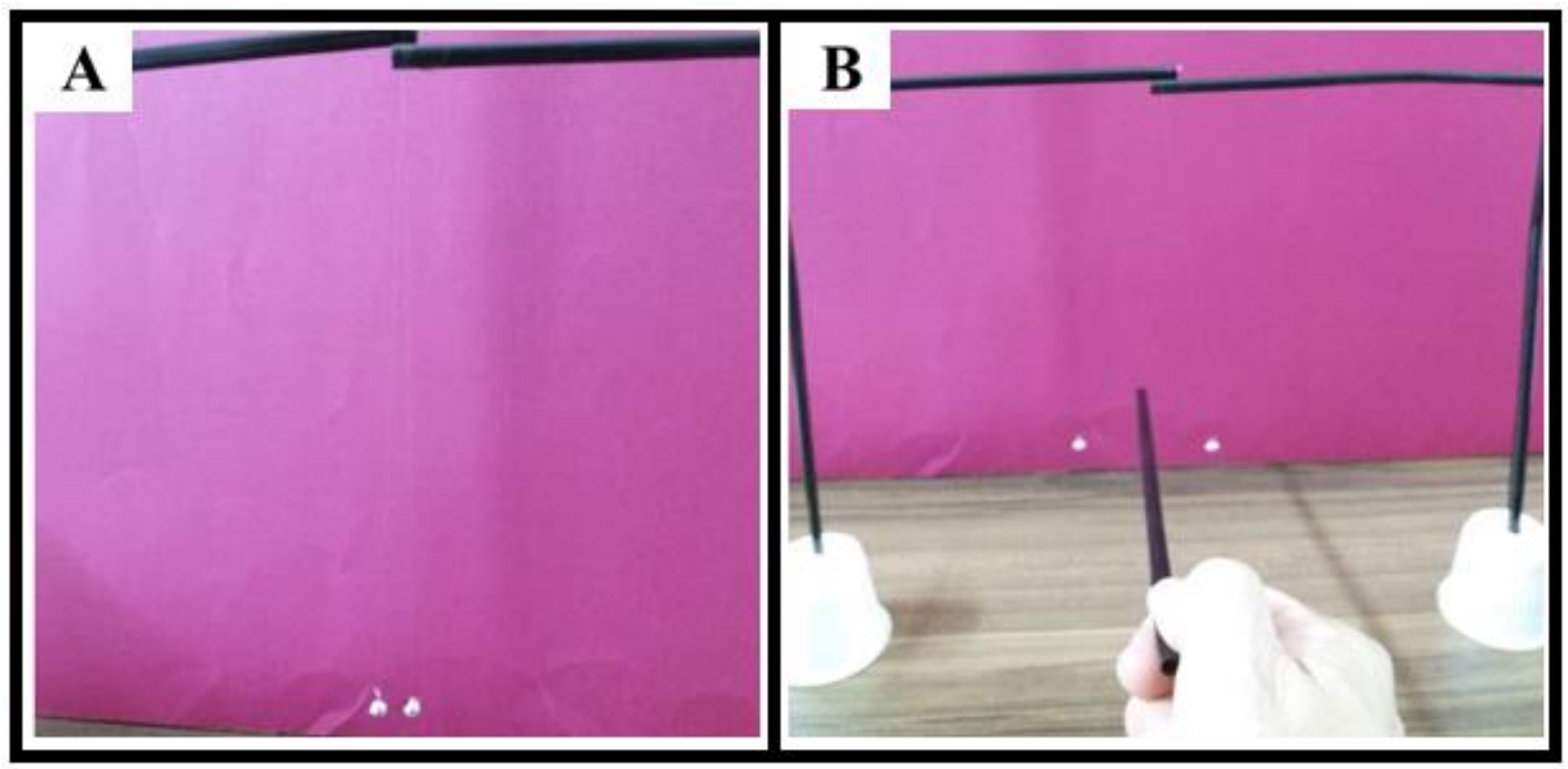

Fig. 1 - A: Foto das miçangas eletrizadas e ligeiramente afastadas; B - Foto das miçangas eletrizadas com maior afastamento por ação do canudinho eletrizado.

Fonte: Autores.

É preciso alertar que os ângulos, caso sejam medidos diretamente sobre o equipamento, devem ser determinados com bastante cuidado para que não sejam alterados por causa da interferência do transferidor ou das mãos do experimentador que seguram seja o transferidor, seja o canudinho. Um procedimento alternativo que aqui foi realizado para eliminar tais interferências é o de fotografar os afastamentos dos fios em cada situação e com um celular medir os ângulos nas imagens com um transferidor posto sobre sua tela.

\section{Questões teóricas}

\section{1 Aproximações e idealizações}

Para os cálculos apresentados a seguir, algumas aproximações são feitas: 1) por serem diminutas, as miçangas de material isolante, mas com metálico filme fino superficial, podem ser aproximadas, em termos de seus comportamentos eletrostáticos aqui envolvidos, como condutoras; 2) suas dimensões muito próximas as qualificam com mesmas capacitâncias. Por consequência, após o toque do canudinho eletrizado nas miçangas redondas inicialmente neutras, permite-se idealizar que: 3) a distribuição de cargas sobre suas superfícies é esférica; 4) suas quantidades de cargas (Q) se equivalem; 5) considera-se as dimensões do canudinho como infinita no comprimento e desprezível no diâmetro, suposições que são cabíveis dadas as proporções das medidas envolvidas dos objetos em interação; 6) assume-se que 
o processo de eletrização por atrito no canudinho produza distribuição de cargas uniforme sob sua superfície.

\section{2 Cálculos e medidas}

Na situação em que as miçangas eletrizadas se repelem entre si, é possível encontrar suas cargas individuais $(\mathrm{Q})$ utilizando a medida do ângulo $(\theta)$ de separação entre os fios e seu comprimento $(l)$, conforme a equação 1, deduzida na Fig. 2.

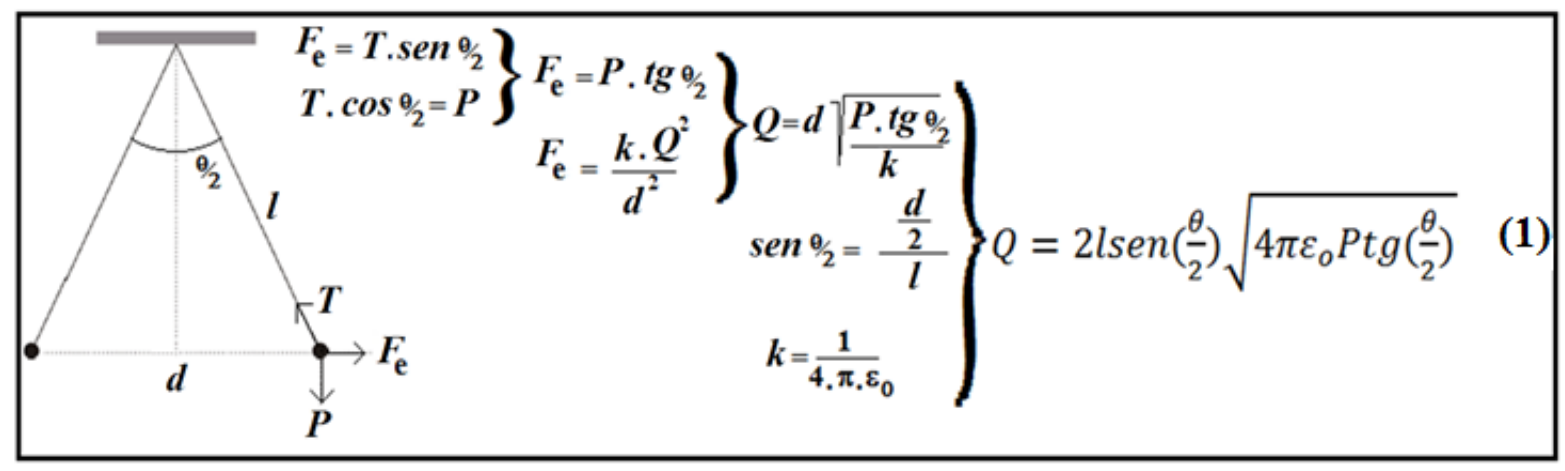

Fig. 2 - Diagrama de forças e equação 1. Fonte: autores.

Assim sendo, um transferidor ajustado sobre a tela do celular com a foto indicada na Fig. 1A indicou o ângulo de afastamento próximo de $3^{\circ}$ para um comprimento $l$ de $0,15 \mathrm{~m}$. Já as miçangas tiveram as massas obtidas por meio de balança analítica ${ }^{3}$ com quatro casas de precisão e não apresentaram diferenças significativas, cujos valores foram de $2,97 \times 10^{-5} \mathrm{~kg}$ e $2,98 \times 10^{-5} \mathrm{~kg}$. Considerando as massas iguais num valor médio, para efeitos de cálculos ainda se tem que $1 / 4 \pi \varepsilon_{0}=9 \times 10^{9}\left(\mathrm{~N} \cdot \mathrm{m}^{2} / \mathrm{C}^{2}\right)$ e que o peso $(P)$ das miçangas se obtém pelo produto entre massa e aceleração da gravidade $\left(g=9,8 \mathrm{~m} / \mathrm{s}^{2}\right)$. Por tais valores, o cálculo da equação 1 nos permite encontrar o valor de $Q=2,3 \times 10^{-10} \mathrm{C}$.

Feito isto, tem-se pela lei de Gauss que a relação entre o campo elétrico $(E)$ e a densidade linear de carga ( $\lambda$ ) (HALLIDAY, RESNICK, 1984, p. 51) é:

$$
E=\frac{\lambda}{2 \pi \epsilon_{0} d^{\prime}}
$$

Sendo que $d^{\prime}$ trata-se da distância do canudinho a uma das cargas (Q) repelidas e, partindo-se do novo equilíbrio de forças $\left[F_{E}=Q . E=P \operatorname{tg}\left(\theta^{\prime} / 2\right)\right]$, do valor das cargas $Q$ determinadas acima, e de que $d^{\prime}=l \cdot \operatorname{sen} \theta^{\prime} / 2$, a densidade linear $(\lambda)$ fica dada pela equação 2 .

$$
\lambda=2 \pi \epsilon_{0} l Q^{-1} P \operatorname{sen}\left(\frac{\theta^{\prime}}{2}\right) \operatorname{tg}\left(\frac{\theta^{\prime}}{2}\right)
$$

\footnotetext{
${ }^{3}$ Instrumento comum nos laboratórios das instituições.
} 
Em que $\theta^{\prime}$ é o novo ângulo entre as cargas devido à repulsão do canudinho eletrizado, sendo, no caso da Fig. 1B, de aproximadamente $20^{\circ}$, o que permite encontrar o valor de $\lambda=$ $3,25 \times 10^{-7} \frac{\mathrm{C}}{\mathrm{m}}$.

Conforme mostram os cálculos e uma vez sabido o valor das massas das miçangas empregadas, as medições que os estudantes devem proceder são tão somente dos ângulos entre os fios das miçangas eletrizadas antes e após a colocação do canudinho eletrizado, respectivamente, $\theta$ e $\theta^{\prime}$, e o comprimento do fio de nylon $l$. Todavia, é preciso dizer que embora os valores aqui divulgados sejam tipicamente obtidos neste experimento, uma variação pode ocorrer dependendo dos materiais usados e da umidade do ar do dia da experiência.

\section{3 Uma reflexão acerca da consideração do canudo com comprimento finito}

A equação do campo elétrico $E$ de uma barra (canudo) de comprimento finito $x$ a uma distância $d$ ' de seu centro geométrico é dada por:

$$
E=\frac{1}{4 \pi \varepsilon_{0}}\left(\frac{\lambda x}{d^{\prime} \sqrt{\left(\frac{x}{2}\right)^{2}+d^{\prime 2}}}\right)
$$

Para alunos de graduação, os detalhes da obtenção da equação acima ${ }^{4}$ podem ser fornecidos sem maiores problemas, mostrando a solução de integrais (SEARS; ZEMANSKY; 2004, p. 19). Para alunos do ensino médio, esta pode ser apresentada de forma direta. Trabalhar esta expressão é interessante porque torna possível comprovar se a consideração de um canudo com comprimento infinito é satisfatória. Neste caso, a miçanga funciona como uma carga de prova para o campo elétrico do canudo e, ao se isolar $\lambda$, substituindo $E$ por $\left[(P / Q) \cdot \operatorname{tg}\left(\theta^{\prime} / 2\right)\right]$ e $d^{\prime}$ por $\left(l . s e n \theta^{\prime} / 2\right)$, chega-se à seguinte equação para a densidade linear de cargas para o canudo de comprimento $x$ finito:

$$
\lambda=\frac{4 \pi \varepsilon_{0}}{Q} P l \operatorname{sen}\left(\frac{\theta^{\prime}}{2}\right) \operatorname{tg}\left(\frac{\theta^{\prime}}{2}\right)\left[\frac{1}{x} \sqrt{\left(\frac{x}{2}\right)^{2}+l \operatorname{sen}^{2}\left(\frac{\theta^{\prime}}{2}\right)}\right]
$$

Cabe notar que se $x$ for muito grande, tendendo a infinito $(x \rightarrow \infty)$, a expressão entre colchetes se reduz a 0,5 e se obtém a equação 2 , cujo resultado para $\lambda$ já foi mencionado. Todavia, considerando o comprimento típico de um canudo de refresco com $x=25 \mathrm{~cm}$ ou 0,25 $\mathrm{m}$, é importante discutir que neste cálculo não se utiliza o valor de toda a extensão do canudo, pois se deve considerar apenas a região eletrizada em que o atrito entre o canudo e o papel é estabelecido. É necessário descontar a parte do canudo utilizada para segurá-lo, pois o experimentador está conectado nesta parte e, portanto, não pode ser contabilizada para o cálculo da distribuição de cargas total do canudo. Pela foto apresentada na Fig. 1B, são cerca de $3 \mathrm{~cm}$ do comprimento do canudo para segurá-lo. Logo, tem-se uma barra (canudo) de $x=0,22 \mathrm{~m}$ para

\footnotetext{
${ }^{4}$ Sabendo que $\lambda . x$ equivale à carga total $Q$ ’ do canudo.
} 
o cálculo de $\lambda$. E, com os valores de $\theta^{\prime}=20^{\circ}$ e $l=0,15 \mathrm{~m}$, a expressão entre colchetes da equação 3 fornece o valor numérico aproximado de $0,5138 \approx 0,5$. Este resultado é interessante e mostra com excelente aproximação que o canudo realmente pode ser considerado como uma barra infinita com relação às miçangas. Enfim, a aproximação 5 (Aproximação e idealizações) anteriormente mencionada e que fora então realizada pode ser assim provada por meio do modelo matemático que considera o canudo como uma barra de comprimento finito.

\section{Conclusão}

O trabalho descreveu um experimento simples e com materiais acessíveis que pode ser empregado pelo professor em sala de aula com o objetivo de aplicar conceitos de eletrostática estudados em nível ensino médio e superior. Ao dirigir-se para a sala de aula com o equipamento previamente montado pelo estudante, a realização experimental dispende reduzido tempo para os procedimentos e retirada de medidas. Assim, a atividade experimental proposta pode se juntar a exercícios de aplicação da teoria, complementando-os com um modo de representação diferenciado. Em função disso, instigam-se capacidades cognitivas suplementares às escolares e, consequentemente, aumentam-se as possibilidades de maior significação dos conceitos ensinados.

\section{Referências}

HALLIDAY, D.; RESNICK, R. Física 3. 4. ed. Rio de Janeiro e São Paulo: Livros Técnicos e Científicos Editora, 1984.

KENDON, A. Gesture: visible action as utterance. Cambridge: Cambridge University Press, 2005.

KIM, M.; ROTH, W.-M.; THOM, J. Children's gestures and the embodied knowledge of geometry. International Journal of Science and Mathematics Education, v. 9, p. 207-238, 2011.

LABURÚ, C. E.; SILVA, O. H. M. O Laboratório didático a partir da perspectiva da multimodalidade representacional. Ciência \& Educação, Bauru, v. 17, n. 3, p. 721-734, 2011.

LABURÚ, C. E.; SILVA, O. H. M; BARROS, M. A Para-raios: um experimento simples e de baixo custo para a eletrostática. Caderno Brasileiro de Ensino de Física, Florianópolis, v. 25 , n. 1, 168-182; 2008.

LABURÚ, C. E.; SILVA, O. H. M; FORÇA, A. C. Acurácia na retirada da medida instigada por uma estratégia de ensino de orientação kuhniana. Revista Brasileira de Ensino de Física, v. 34, n. 2, p. 2503-2503-6, 2012. 
LABURÚ, C. E.; SILVA, O. H.; M.; ZÔMPERO, A. F. Significados de eletrostática interpretados por meio da gesticulação de estudantes. Ciência \& Educação, Bauru, v. 21, n. 4, p. 851-867, 2015.

McNEILL, D. Gesture and thought. Chicago: David McNeil, 2005. 318 p.

MILLAR, R. Towards a role for experiment in the science teaching laboratory. Studies in Science Education, v. 14, p. 109-118, 1987.

PRAIN, V.; WALDRIP, B. An exploratory study of teachers' and students' use of multimodal representations of concepts in primary science. International Journal of Science Education, v. 28, n. 15, p. 1843-1866, 2006.

SEARS; ZEMANSKY. Física III: eletromagnetismo. São Paulo: Pearson Addison Wesley, 2004. 